\title{
AMIGO2 mRNA expression in hippocampal CA2 and CA3a
}

\author{
Annelies Laeremans · Julie Nys • Walter Luyten • \\ Rudi D'Hooge $\cdot$ Melissa Paulussen $\cdot$ Lut Arckens
}

Received: 3 December 2011 / Accepted: 17 January 2012

(C) Springer-Verlag 2012

\begin{abstract}
AMIGO2, or amphoterin-induced gene and ORF (open reading frame) 2, belongs to the leucine-rich repeats and immunoglobulin superfamilies. The protein is a downstream target of calcium-dependent survival signals and, therefore, promotes neuronal survival. Here, we describe the mRNA distribution pattern of AMIGO2 throughout the mouse brain with special emphasis on the hippocampus. In the Ammon's horn, a detailed comparison between the subregional mRNA expression patterns of AMIGO2 and Pcp4 (Purkinje cell protein 4)—a known molecular marker of hippocampal CA2 (Cornu Ammonis 2)-revealed a prominent AMIGO2 mRNA expression level in both the CA2 and the CA3a (Cornu Ammonis 3a) subregion of the dorsal and ventral hippocampus. Since this CA2/CA3a region is particularly resistant to neuronal injury and neurotoxicity [Stanfield and Cowan (Brain Res 309(2):299-307 1984); Sloviter (J Comp Neurol 280(2):183-196 1989); Leranth and Ribak (Exp Brain Res 85(1):129-136 1991); Young and Dragunow (Exp Neurol 133(2):125-137 1995); Ochiishi et al. (Neurosci 93(3):955-967 1999)], we suggest that the expression pattern of AMIGO2 indeed fits with its involvement in neuroprotection.
\end{abstract}

\footnotetext{
A. Laeremans $\cdot$ J. Nys $\cdot$ M. Paulussen $\cdot$ L. Arckens $(\bowtie)$

Laboratory of Neuroplasticity and Neuroproteomics,

University of Leuven, Naamsestraat 59,

Box 2467, 3000 Leuven, Belgium

e-mail: Lut.Arckens@bio.kuleuven.be

W. Luyten

Biomedical Sciences Group, Department of Woman and Child,

University of Leuven, Herestraat 49, 3000 Leuven, Belgium

R. D'Hooge

Laboratory of Biological Psychology, University of Leuven,

Tiensestraat 102, 3000 Leuven, Belgium
}

Keywords AMIGO2 - Mouse · Hippocampus · In situ hybridization

\section{Introduction}

A few years ago, the genes AMIGO2, Alivin1 and DEGA (differentially expressed in gastric adenocarcinomas) were independently identified by three different research groups (Kuja-Panula et al. 2003; Ono et al. 2003; Rabenau et al. 2004). Kuja-Panula and colleagues (2003) discovered a new gene that was induced by the neurite-outgrowthpromoting factor amphoterin. Therefore, this gene was called amphoterin-induced gene and ORF, also AMIGO. Two related genes, AMIGO2 and AMIGO3, were also sequenced. These three AMIGOs, belonging to the leucinerich repeats (LRRs) and immunoglobulin (Ig) superfamilies, formed a novel family of transmembrane proteins. In that same year, Ono et al. (2003) identified Alivin1 as a new gene that promoted depolarization-dependent survival of cerebellar granule neurons. Again the presence of two related genes was noticed, namely Alivin2 and Alivin3, and it was suggested that mutations of Alivin1 could cause neurodegenerative diseases like Alzheimer's and Parkinson's disease. Only recently, haploinsufficiency of AMIGO2 has also been reported to be potentially responsible for mental retardation (Gimelli et al. 2011). Rabenau et al. (2004) found a gene that was differentially expressed in $45 \%$ of all human gastric adenocarcinomas that they named differentially expressed in gastric adenocarcinomas, or DEGA. Alivin1 and DEGA turned out to be identical to AMIGO2. Analysis of RT-PCR results on a variety of mouse tissues indicated that AMIGO2 mRNA was preferentially located in the central nervous system (Kuja-Panula et al. 2003). By means of immunocytochemistry, Ono et al. 
(2003) demonstrated the presence of AMIGO2 protein in the granular and Purkinje cell layers of the cerebellum, and described an AMIGO2-enriched region between CA1 and $\mathrm{CA} 3$ in the rat hippocampus.

The hippocampus contains two main groups of neurons: granule and pyramidal cells localized in the dentate gyrus (DG) and the Ammon's horn (Cornu Ammonis, CA), respectively. The latter can be subdivided in the CA1, CA2 and CA3 subfield (Lorente dé No 1934; Hunsaker et al. 2008), next to the hilus of the dentate gyrus (Amaral 1978). Each hippocampal region can be distinguished on the basis of morphological, connectional or electrophysiological properties and susceptibility to insults (Lein et al. 2004; Mercer et al. 2007). Recently, the anatomical boundaries of the different hippocampal subregions have been defined based on the highly restricted and non-overlapping expression patterns of a certain set of genes. For example, the molecular marker alpha mannosidase 1 is only expressed in CA1, while bcl-2-related ovarian killer protein is only present in CA3 and Pcp4 expression is particularly pronounced in CA2 and the dentate gyrus (Zhao et al. 2001; Lein et al. 2004, 2005).

In this study, we report the mRNA distribution pattern of the fairly recently discovered and functionally intriguing AMIGO2 gene along both the anterioposterior and mediolateral axis throughout the whole mouse brain, and specifically focus on its peculiar expression pattern in the Ammon's horn of the hippocampus. Based on the molecularly determined cytoarchitectural boundaries, we initially hypothesized that AMIGO2 was especially enriched in hippocampal CA2, the only subregion of the Ammon's horn characterized by Pcp4 mRNA expression. After careful examination and comparison of the AMIGO2 and Pcp4 expression patterns, we provide compelling evidence that AMIGO2 expression is present but not confined to CA2, since this gene is also enriched in the distal part of the $\mathrm{CA} 3$ region, i.e., CA3a.

\section{Materials and methods}

Animals and tissue preparation

Adult male C57Bl/6J mice (age $\geq 3$ months) were obtained from Janvier Elevage (Le Genest-St-Isle, France) and housed under standard laboratory conditions. They were raised in an 11/13 $\mathrm{h}$ dark/light cycle with food and water ad libitum. All experiments have been approved by the Institutional Laboratory Animal Use and Care Committee (Animal Facilities, KU Leuven, Belgium) and were conducted in strict accordance with the European Communities Council Directive of 22 September 2010 (2010/63/ $\mathrm{EU})$.
Animals $(n=6)$ were killed by cervical dislocation, brains were rapidly removed, immediately frozen in 2-methylbutane (Merck, Overijse, Belgium) at a temperature of $-40^{\circ} \mathrm{C}$ and stored at $-70^{\circ} \mathrm{C}$ until sectioning. Twenty-five micrometer thick coronal and sagittal sections were cut on a cryostat (Microm HM 500 OM, Walldorf, Germany), mounted on $0.1 \%$ poly-L-lysine (SigmaAldrich, St. Louis, MO)-coated glass slides and stored at $-30^{\circ} \mathrm{C}$ until further processing.

In situ hybridization

In situ hybridization was performed with synthetic oligonucleotide probes (Eurogentec, Seraing, Belgium) with sequence $5^{\prime}$-gagccctgaaggacacggcggccggacagaatggcaaag tcaag- $3^{\prime}$ for AMIGO2 and sequence $5^{\prime}$-gacaaagtgccggagc gaccaacggaaaagacaagacgtcaggag- $3^{\prime}$ for Pcp4. As described earlier (Arckens et al. 1995; Cnops et al. 2007), each probe was $3^{\prime}$-end labeled with ${ }^{33} \mathrm{P}$-dATP using terminal deoxynucleotidyl transferase (Invitrogen, Merelbeke, Belgium). Unincorporated nucleotides were separated from the labeled probe with miniQuick SpinTM Oligo Columns (Roche Diagnostics, Brussels, Belgium). Prior to hybridization for AMIGO2 and Pcp4 mRNA, adjacent sections were fixated, dehydrated and delipidated. The radioactively labeled probe was added to a hybridization cocktail (50\% formamide, $4 \times$ standard saline sodium citrate buffer, $1 \times$ Denhardt's solution, $1 \%$ sarcosyl $N$-lauroyl sarcosine sodium salt, $20 \mathrm{mM} \mathrm{NaHPO}$. $\mathrm{pH} 7.4,10 \%$ dextran sulfate, $100 \mu \mathrm{g} / \mathrm{ml}$ salmon sperm DNA, $250 \mu \mathrm{g} / \mathrm{ml}$ tRNA) and applied to the cryostat sections $\left(10^{6}\right.$ counts per section) for an overnight incubation at $37^{\circ} \mathrm{C}$ in a humid chamber. As a control, a 100-fold excess of unlabeled probe was added to the hybridization cocktail under identical conditions. This competition experiment abolished the signal generated by normal antisense probe hybridization (data not shown). The following day, sections were rinsed in $1 \times$ standard saline sodium citrate buffer at a temperature of $42^{\circ} \mathrm{C}$, dried and exposed to an autoradiographic film during a period of 3 weeks for AMIGO2 and 4 days for Pcp4 (Biomax MR, Kodak, Zaventem, Belgium). Afterwards the films were developed in Kodak D19 developing solution and fixed in rapid fixer (Ilford Hypam, Kodak). The autoradiograms were converted to digital images by means of a scanner (CanoScan LIDE 600F, Canon). Pseudo-colored overlays of the AMIGO2 (pink) and Pcp4 (green) digital autoradiograms were created in Adobe Photoshop (version CS5, Adobe).

\section{Histology}

Histology was performed to assist the interpretation of distribution patterns obtained by in situ hybridization. 
Sections were Nissl-stained (Cresyl violet $1 \%$, Fluka Chemika, Sigma-Aldrich) according to standard procedures.

\section{Results}

AMIGO2 distribution throughout the mouse brain

We examined the distribution pattern of AMIGO2 mRNA in coronal and sagittal sections covering the entire mouse brain, with special emphasis on the hippocampal subregions, as defined by the most recent stereotaxic atlas (Franklin and Paxinos 2008). Overall, AMIGO2 mRNA was expressed in a minority of brain regions. In a series of coronal sections along the anterioposterior axis, a clearly delineated pronounced expression level was located in the mitral cell layer of the olfactory bulb and the granular cell layer of the accessory olfactory bulb (Fig. 1a), the preoptic area (Fig. 1b), the habenula (Fig. 1c), premammillary nuclei (Fig. 1d), the hippocampus (Fig. 1c, d) and the cerebellum (Fig. 1e).

\section{AMIGO2 mRNA expression in the hippocampus}

Both in serial coronal and sagittal sections, a highly specific and intense AMIGO2 mRNA signal could be discerned in the Ammon's horn flanked by CA1 and CA3. Because of this remarkable expression, we decided to compare this AMIGO2 mRNA expression pattern with that of a protein known to be specifically expressed in CA2, namely Pcp4. In Fig. 2, a series of consecutive coronal brain sections spanning the entire hippocampus is shown, analyzed for either AMIGO2 (Fig. 2, middle column) or Pcp4 (Fig. 2, left column) mRNA expression. With regard to the hippocampus, the distribution of Pcp4 mRNA was very dense in the dentate gyrus, the CA2 subregion and the fasciola cinerea. This latter structure emerges rostrally at the midline because the CA2 subfield splits into two separated regions by the insertion of CA1 at these Bregma levels (Fig. 2, rows b, c, arrowheads). In contrast, the CA1 and CA3 subfields were completely devoid of Pcp4 signal. Apart from the DG, AMIGO2 displayed a rather similar hippocampal distribution pattern as Pcp4.

In the right column of Fig. 2 and in Fig. 3, pseudocolored overlays of AMIGO2 (pink) and Pcp4 (green) mRNA expression are shown for different Bregma levels. A detailed comparison of AMIGO2 and Pcp4 mRNA distribution patterns illustrates an overlap of expression in CA2. However, while Pcp4 mRNA expression was restricted to $\mathrm{CA} 2$, the $\mathrm{AMIGO} 2$ mRNA signal clearly extended further into the CA3 subfield, comprising CA3a in both the dorsal and the ventral horn (Figs. 2, 3; Ochiishi et al. 1999). a

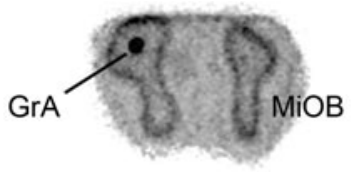

b
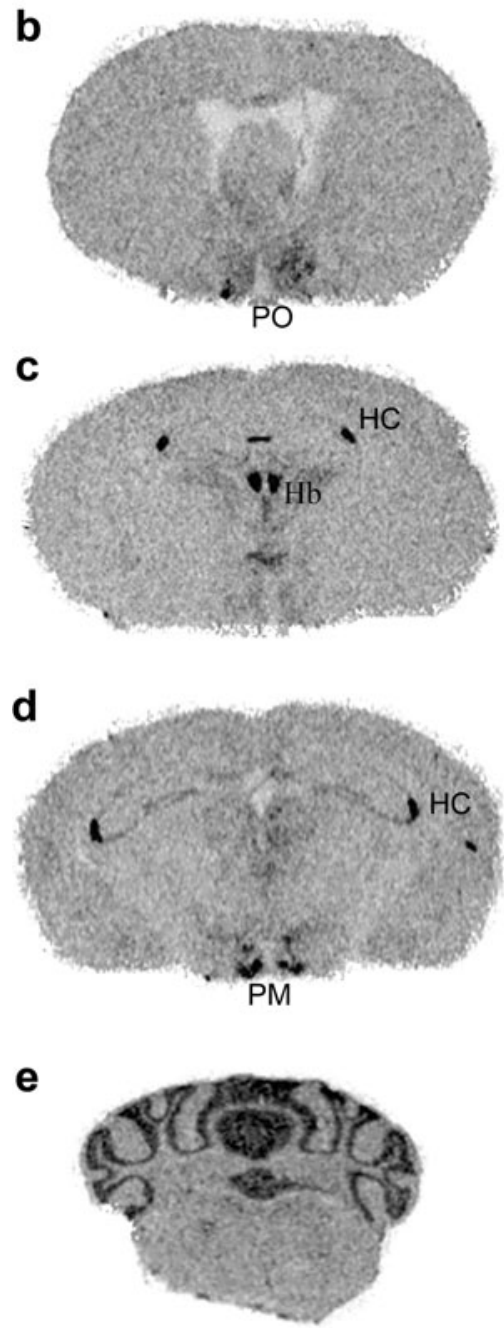

Fig. 1 AMIGO2 mRNA distribution in the mouse brain. Five different Bregma levels are shown along the anterior to posterior axis: a Bregma $3.92 \mathrm{~mm}$, b Bregma $0.02 \mathrm{~mm}$, c Bregma $-1.82 \mathrm{~mm}$, d Bregma $-2.46 \mathrm{~mm}$ and e Bregma $-6.72 \mathrm{~mm}$. AMIGO2 mRNA expression could be observed in the mitral cell layer of the olfactory bulb and the granular cell layer of the accessory olfactory bulb (a), the preoptic area (b), the habenula $(\mathbf{c})$, the premammillary nuclei $(\mathbf{d})$, the hippocampus (c, d) and the cerebellum (e). $G r A$ granular cell layer accessory olfactory bulb, $H b$ habenula, $H C$ hippocampus, $M i O B$ mitral cell layer olfactory bulb, $P M$ premammillary nucleus, $P O$ preoptic area. Scale bar $2 \mathrm{~mm}$

In sagittal sections, the same distribution patterns could be discerned (Fig. 4). Pcp4 as well as AMIGO2 mRNA was clearly present in CA2 (Fig. 4, arrowheads), but only AMIGO2 mRNA was detected in the CA3a region. In the middle and right columns of row B in Fig. 4, CA3a can be easily distinguished in the Ammon's horn based on high 
a
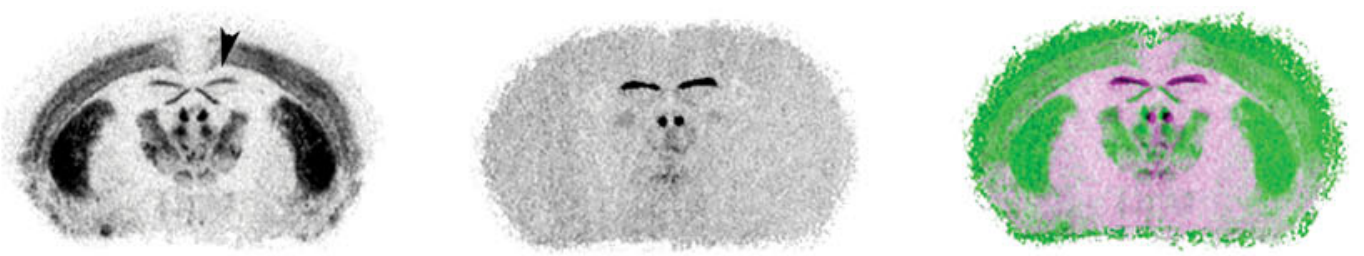

b
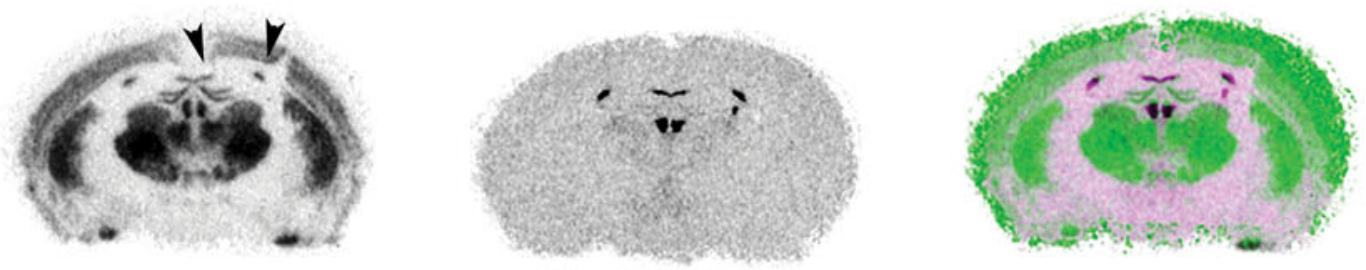

C
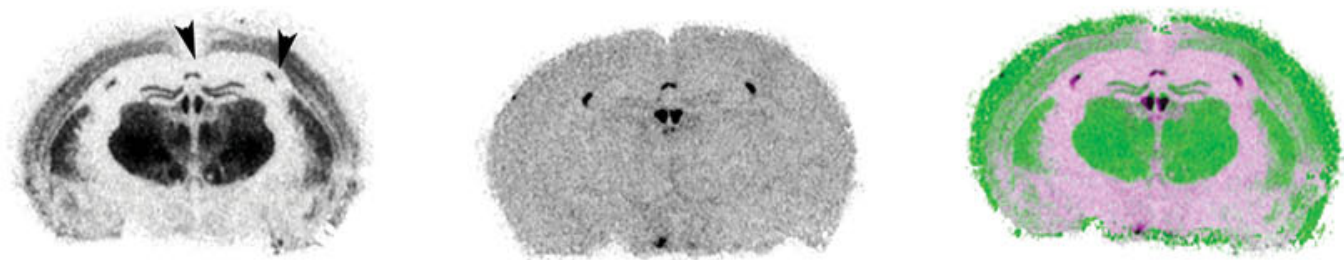

d
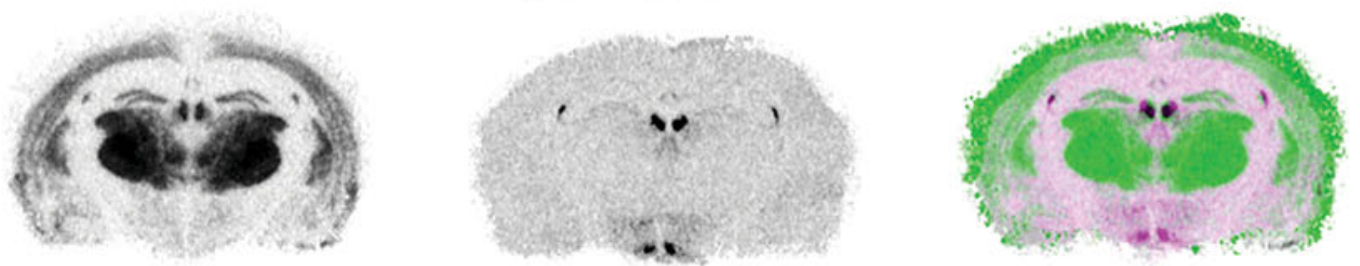

e
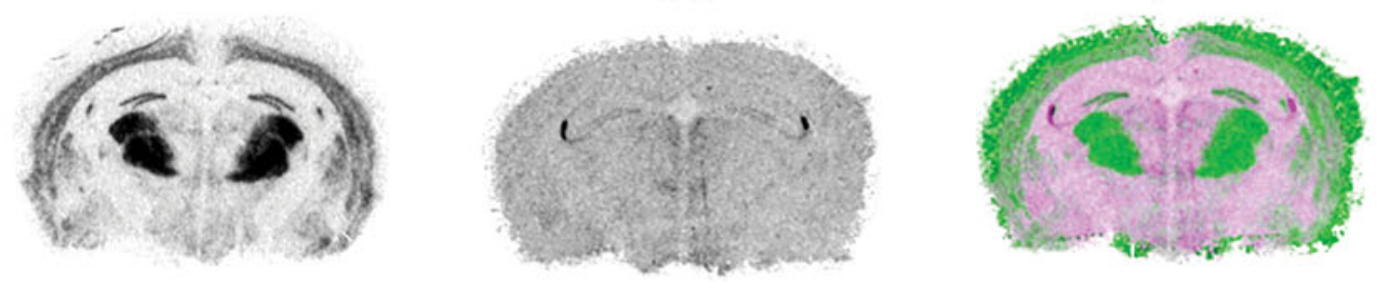

f
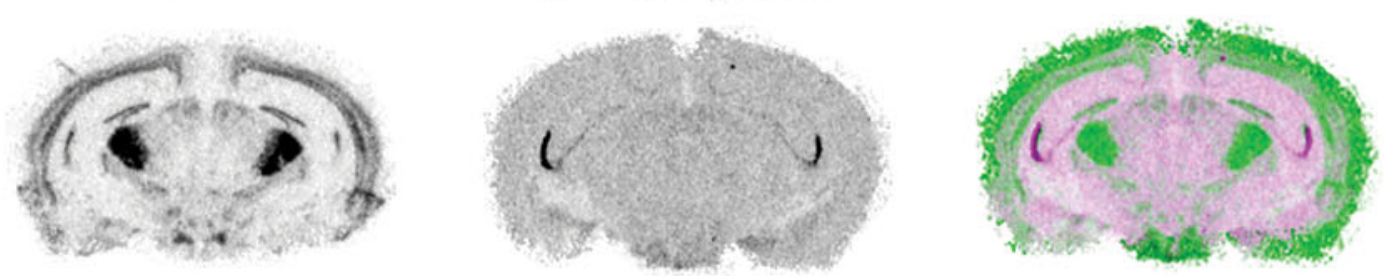

g
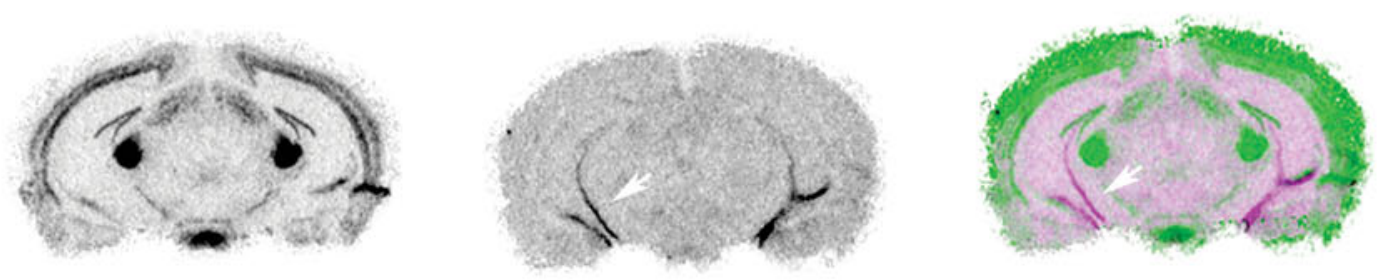

h
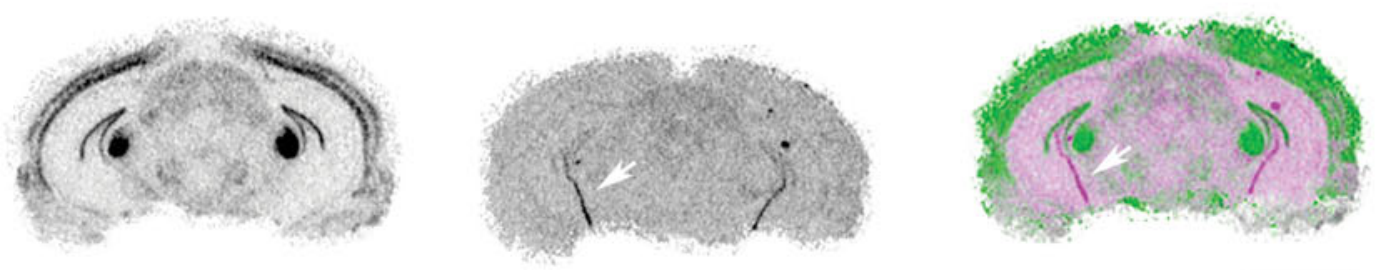
4Fig. 2 AMIGO2 and Pcp4 expression throughout the hippocampus. Series of adjacent coronal brain sections $(150 \mu \mathrm{m}$ interval, from Bregma level $-1.22 \mathrm{~mm}$ in row $\mathbf{a}$ to $-3.16 \mathrm{~mm}$ in row $\mathbf{h}$ ) illustrate Pcp4 and AMIGO2 mRNA expression in the left column and middle column, respectively. In the right column, a pseudo-colored overlay of both sections is shown with AMIGO2 represented in pink and Pcp4 in green. White arrows indicate hippocampal regions solely expressing AMIGO2 (no coinciding Pcp4 expression). Arrowheads indicate the division of CA2 into a separate lateral CA2 region localized between $\mathrm{CA} 1$ and CA3 and the medial fasciola cinerea. Scale bar $3 \mathrm{~mm}$

AMIGO2 mRNA expression levels (Fig. 4, row b, middle column, white arrows), whereas the Pcp4 signal was clearly absent in this hippocampal subdivision (Fig. 4, row b, left column).

\section{Discussion}

In this study we analyzed the distribution pattern of AMIGO2 mRNA in the mouse brain, with special emphasis on the hippocampus. In view of the enriched AMIGO2 mRNA expression in what appeared to be CA2, we compared the hippocampal mRNA expression of AMIGO2 with that of Pcp4. Pcp4 was previously used as a molecular marker to demarcate hippocampal CA2 by Lein and colleagues $(2004,2005)$, given that they had found discrepancies between the neuroanatomical delineation of the CA subregions based on Pcp4 and those depicted in the Paxinos and Franklin brain atlas (2001). Our delineation of CA2 determined by in situ hybridization for Pcp4 showed that this region emerged at the midline of the anterior hippocampus. Slightly more posterior, this CA2 subregion became separated into the medially located fasciola cinerea and the laterally remaining $\mathrm{CA} 2$ subregion by the insertion of CA1. In contrast, the brain atlas designated the region at the midline, which according to Lein et al. (2005) and our observations was CA2, to be CA1 (Paxinos and Franklin 2001, Fig. 4, Bregma $-1.22 \mathrm{~mm}$ ). Only recently, in the new edition of the mouse brain atlas the delineation of CA2
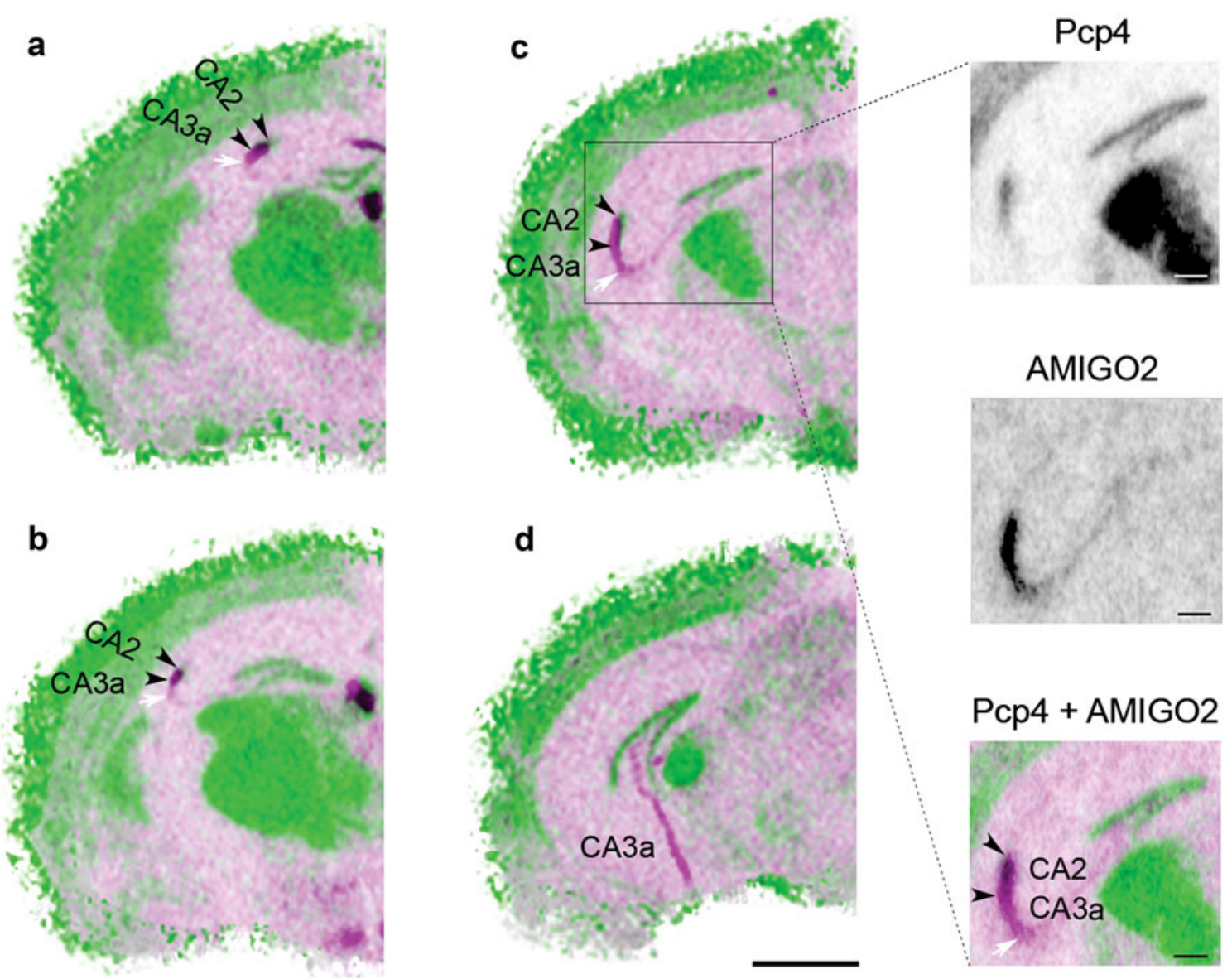

Fig. 3 AMIGO2-Pcp4 pseudo-colored overlays display the presence of AMIGO2 beyond CA2 into CA3a. AMIGO2 is represented in pink and Pcp4 in green on four different Bregma levels. a $-1.34 \mathrm{~mm}$, b $-1.94 \mathrm{~mm}, \mathbf{c}-2.54 \mathrm{~mm}$ and $\mathbf{d}-3.16 \mathrm{~mm}$ relative to Bregma. High-resolution magnifications of the hippocampal region indicated with a box in $\mathbf{c}$ are shown in the right column: upper Pcp4 mRNA distribution, middle AMIGO2 mRNA distribution and lower pseudocolored overlay of AMIGO2 and Pcp4. Arrowheads delineate CA2 and white arrows demarcate the lateral extent of CA3a. CA2 Cornu Ammonis 2, CA3a Cornu Ammonis 3a. Scale bar a-d $1.5 \mathrm{~mm}$, scale bar magnification images $2 \mathrm{~mm}$ 
a

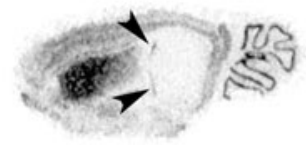

b

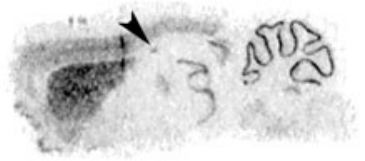

C

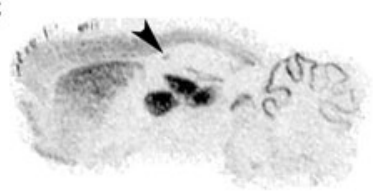

d
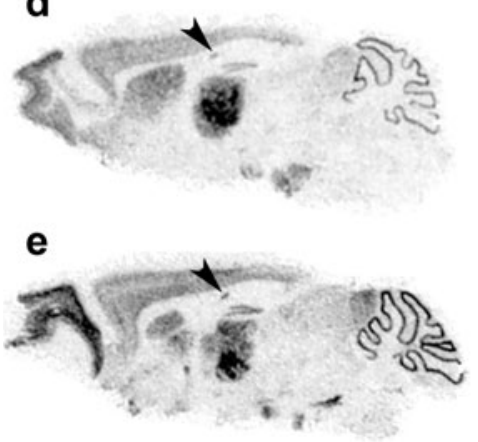
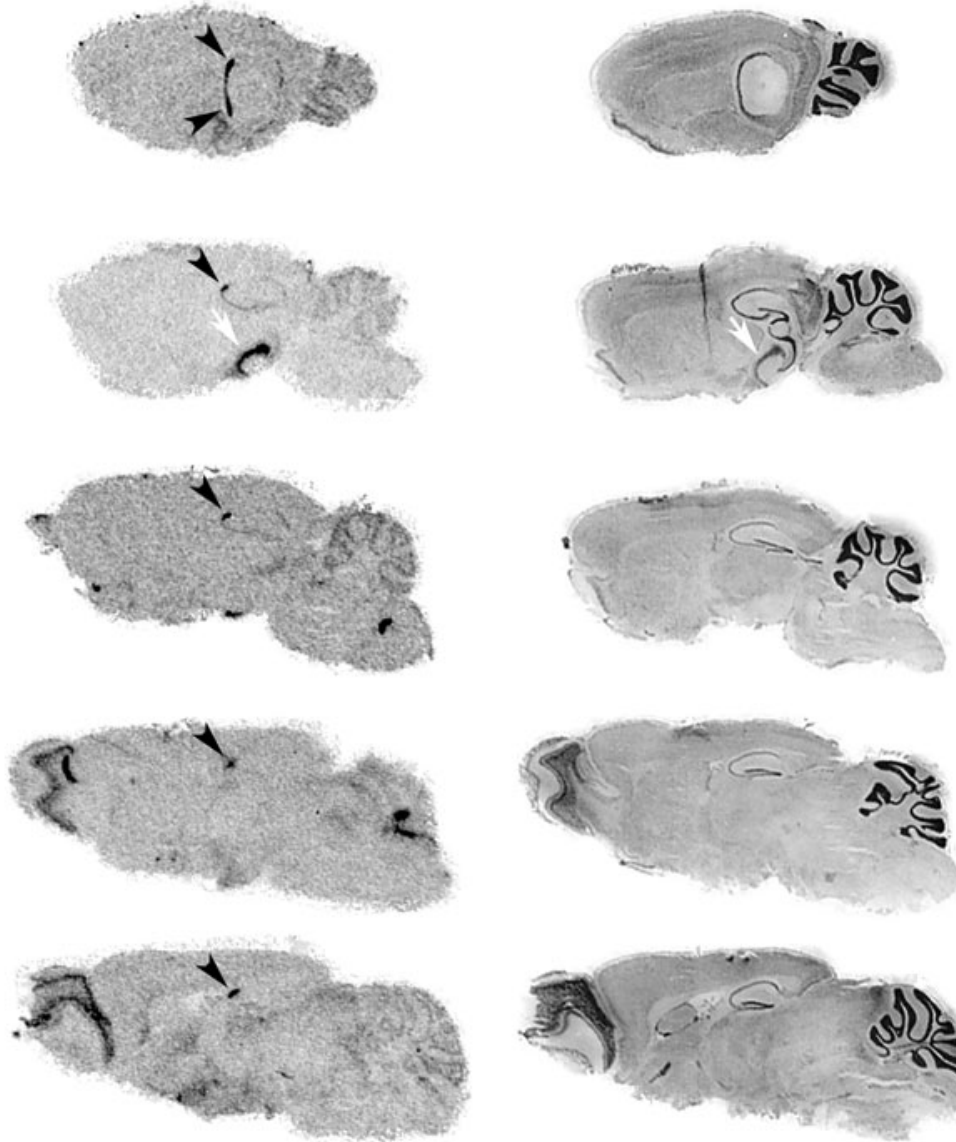
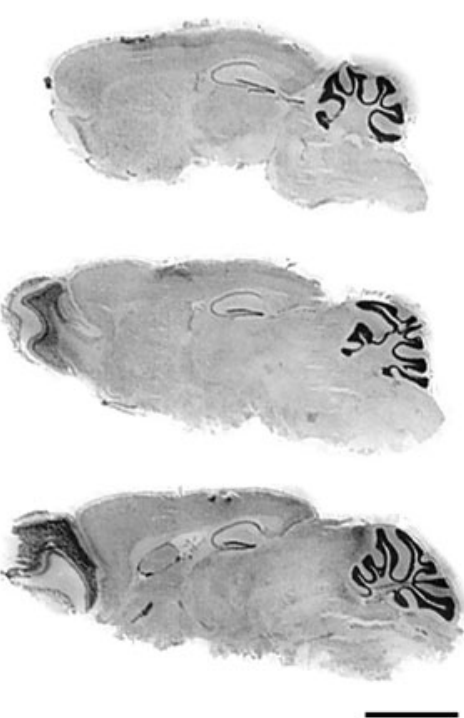

Fig. 4 AMIGO2 and Pcp4 expression in serial, sagittal sections throughout the hippocampus. A series of adjacent sagittal brain sections between M-L 3.25 and $1.32 \mathrm{~mm}$ is visualized for Pcp4 ISH in the left column, for AMIGO2 ISH in the middle column and for Niss1

has been adjusted (Franklin and Paxinos 2008, Fig. 4, Bregma $-1.22 \mathrm{~mm}$ ) and now corresponds well to our results and those of Lein et al. (2005), confirming the validity of Pcp4 as a marker of CA2 (Zhao et al. 2001; Lein et al. 2004, 2005). This supports the idea that CA2 is a unique region and not just an intermingling of CA1 and CA3 cells. The comparison between AMIGO2 and Pcp4 mRNA distribution showed that, unlike Pcp4, the presence of AMIGO2 mRNA extended beyond CA2 into CA3. Both expression patterns elucidated the exact AMIGO2expressing hippocampal subregion, namely $\mathrm{CA} 2$ and the distal part of CA3 (CA3a). This latter area represents the border region between the hippocampal CA2 and CA3 subfield (Ochiishi et al. 1999).

\section{CA2 and neuronal survival}

It has been suggested that the CA2/CA3a area is relatively resistant to neuronal injury and neurotoxicity. As such, the CA2/CA3a area might be different from other hippocampal staining in the right column. Both AMIGO2 and Pcp4 are located in CA2 (arrowheads), and panels in row b clearly demonstrate the presence of AMIGO2 in the CA3a subregion (white arrows), but the absence of Pcp4. Scale bar $3 \mathrm{~mm}$

CA subfields in its reduced susceptibility to seizureinduced damage during status epilepticus. Previous studies indicated that its resistance to excitotoxic damage could be attributed to the enriched expression of $\mathrm{Ca}^{2+}$-binding proteins-such as parvalbumin and calbindin-that would be able to buffer excessive and neurotoxic $\mathrm{Ca}^{2+}$ influx (Sloviter 1989; Leranth and Ribak 1991; Young and Dragunow 1995). Besides $\mathrm{Ca}^{2+}$-binding proteins, the CA2/ CA3a area is also rich in neurotrophin-3 (NT-3). Vigers et al. (2000) demonstrated that NT-3 expression is especially high in CA2 cells and adjoining CA3 cells at the CA2/CA3 border. In 1994, Cheng and Mattson (1994) indicated that NT-3 can protect cultured hippocampal cells from ischemic damage. Furthermore, the abundance of adenosine A1 receptor-positive neurons in the CA2/CA3a region, which receive direct prominent input from the supramammillary nucleus, could also be linked to protective effects (Stanfield and Cowan 1984; Ochiishi et al. 1999). During epileptic seizures, large quantities of adenosine are released by the cells surrounding the epileptic focus (Winn 
et al. 1980). The released adenosine in combination with high expression of adenosine A1 receptors could prevent seizure propagation in $\mathrm{CA} 2 / \mathrm{CA} 3 \mathrm{a}$, since $\mathrm{A} 1$ receptors inhibit $\mathrm{Ca}^{2+}$ influx and excitatory neurotransmitter release (Fredholm and Hedqvist 1980; Schubert et al. 1986). In humans, Maxwell and colleagues (2003) provided evidence for the differential sensitivity of the different hippocampal subfields to the loss of pyramidal neurons after blunt head injury. In contrast to the other CA subfields, CA2 showed high resistance and accordingly there was no loss of neurons from this subfield. Taken together, these studies infer neuroprotection as a specific characteristic of CA2. As such, the specific expression of AMIGO2 in this hippocampal region could also serve protective functions. Ono et al. (2003) suggested that the expression of Alivin1/ AMIGO2, which is regulated by $\mathrm{Ca}^{2+}$-influx through voltage-dependent L-type calcium channels, inhibits apoptosis and promotes neuronal survival of mouse cerebellar granule cells in response to $\mathrm{Ca}^{2+}$-dependent survival signals. Moreover, the human gene for Alivin1/AMIGO2 was mapped within the same region as Parkinson's disease 8 (PARK8) and familial Alzheimer's disease type 5 (AD5). Consequently to its role as a neuronal survival-promoting gene, mutations of Alivin1/AMIGO2 could contribute to the neurodegenerative mechanisms in Alzheimer's or Parkinson's disease (Ono et al. 2003) and could eventually be a possible target for neuroprotective therapies. Also, the CA2 subfield of the hippocampus was shown to be relatively resistant to neurofibrillary tangles in Alzheimer's disease (Ishizawa et al. 2002).

\section{CA2 and social behavior}

Knockout and pharmacological studies for the CA2-enriched vasopressin $1 \mathrm{~b}$ receptor (Avpr1b) implicate that a lack of Avpr1b leads to reduced forms of olfactory-based social behavior, including social forms of aggression, social recognition memory and social motivation, but intact spatial memory (Caldwell et al. 2008; Jones and McHugh 2011; Stevenson and Caldwell 2011). The hippocampal CA2 subfield is uniquely positioned in this context because of its direct input from both layers II and III from the medial entorhinal cortex via the perforant path (Jones and McHugh 2011), a cortical region involved in the processing of individual social odors (Petrulis et al. 2005). It was, therefore, hypothesized that Avpr1b-enriched CA2 is engaged in the formation or retrieval of memories characterized by an olfactory-based social component (Young et al. 2006; Caldwell et al. 2008; Stevenson and Caldwell 2011). Although our distribution pattern clearly demonstrates that AMIGO2 expression is not restricted to CA2, its predominant expression in CA2 may hint at an additional role for AMIGO2 in the regulation of social behavior.

\section{Conclusion}

By comparison with the distribution pattern of Pcp4, a molecular marker of $\mathrm{CA} 2, \mathrm{AMIGO} 2$ mRNA expression was allocated to the CA2/CA3a hippocampal region of the mouse brain. Based on its co-distribution with $\mathrm{Ca}^{2+}$ binding proteins, growth factors and A1Rs, all related to neuronal survival signaling, we hypothesize AMIGO2 to confer neuronal-survival capacities to this particular brain region in response to an insult.

Acknowledgments The study was sponsored by the Agency for Innovation through Science and Technology Flanders (IWT Vlaanderen) (SBO IWT 50164), Research Foundation Flanders (FWO Vlaanderen) and the Research Council of the K.U.Leuven (IDO 06/004; OT 09/22), Belgium.

\section{References}

Amaral DG (1978) A golgi study of cell types in the hilar region of the hippocampus in the rat. J Comp Neurol 182(4 Pt 2):851-914

Arckens L, Zhang F, Vanduffel W, Mailleux P, Vanderhaeghen JJ, Orban GA, Vandesande F (1995) Localization of the two protein kinase $\mathrm{C}$ beta-mRNA subtypes in cat visual system. J Chem Neuroanat 8:117-124

Caldwell HK, Wersinger SR, Young WS 3rd (2008) The role of the vasopressin $1 \mathrm{~b}$ receptor in aggression and other social behaviours. Prog Brain Res 170:65-72

Cheng B, Mattson MP (1994) NT-3 and BDNF protect CNS neurons against metabolic/excitotoxic insults. Brain Res 640(1-2):56-67

Cnops L, Hu TT, Eysel UT, Arckens L (2007) Effect of binocular retinal lesions on CRMP2 and CRMP4 but not Dyn I and Syt I expression in adult cat area 17. Eur J Neurosci 25:1395-1401

Franklin KBJ, Paxinos G (2008) The mouse brain in stereotaxic coordinates. Elsevier Science, San Diego

Fredholm BB, Hedqvist P (1980) Modulation of neurotransmission by purine nucleotides and nucleosides. Biochem Pharmacol 29(12): $1635-1643$

Gimelli S, Makrythanasis P, Stouder C, Antonarakis SE, Bottani A, Béna $F$ (2011) A de novo 12q13.11 microdeletion in a patient with severe mental retardation, cleft palate, and high myopia. Eur J Med Genet 54(1):94-96

Hunsaker MR, Rosenberg JS, Kesner RP (2008) The role of the dentate gyrus, CA3a, b, and CA3c for detecting spatial and environmental novelty. Hippocampus 18(10):1064-1073

Ishizawa T, Ko LW, Cookson N, Davias P, Espinoza M, Dickson DW (2002) Selective neurofibrillary degeneration of the hippocampal CA2 sector is associated with four-repeat tauopathies. J Neuropathol Exp Neurol 61(12):1040-1047

Jones MW, McHugh TJ (2011) Updating hippocampal representations: CA2 joins the circuit. Trends Neurosci 34(10):526-535

Kuja-Panula J, Kiiltomäki M, Yamashiro T, Rouhiainen A, Rauvala H (2003) AMIGO, a transmembrane protein implicated in axon tract development, defines a novel protein family with leucinerich repeats. J Cell Biol 160(6):963-973 
Lein ES, Zhao X, Gage FH (2004) Defining a molecular atlas of the hippocampus using DNA microarrays and high-throughput in situ hybridization. J Neurosci 24(15):3879-3889

Lein ES, Callaway EM, Albright TD, Gage FH (2005) Redefining the boundaries of the hippocampal CA2 subfield in the mouse using gene expression and 3-dimensional reconstruction. J Comp Neurol 485:1-10

Leranth C, Ribak CE (1991) Calcium-binding proteins are concentrated in the CA2 field of the monkey hippocampus: a possible key to this region's resistance to epileptic damage. Exp Brain Res 85(1):129-136

Lorente dé No R (1934) Studies on the structure of the cerebral cortex. II. Continuation of the study of the ammonic system. J Psych Neurol 46:113-177

Maxwell WL, Dhillon K, Harper L, Espin J, Macintosh TK, Smith DH, Graham DI (2003) There is differential loss of pyramidal cells from the human hippocampus with survival after blunt head injury. J Neuropathol Exp Neurol 62(3):272-279

Mercer A, Trigg HL, Thomson AM (2007) Characterization of neurons in the CA2 subfield of the adult rat hippocampus. J Neuroscience 27(27):7329-7338

Ochiishi T, Saitoh Y, Yukawa A, Saji M, Ren Y, Shirao T, Miyamoto H, Nakata H, Sekino Y (1999) High level of adenosine A1 receptor-like immunoreactivity in the CA2/CA3a region of the adult rat hippocampus. Neurosci 93(3):955-967

Ono T, Sekino-Suzuki N, Kikkawa Y, Yonekawa H, Kawashima S (2003) Alivin 1, a novel neuronal activity-dependent gene, inhibits apoptosis and promotes survival of cerebellar granule neurons. J Neurosci 23(13):5887-5896

Paxinos G, Franklin KBJ (2001) The mouse brain in stereotaxic coordinates. Elsevier Science, San Diego

Petrulis A, Alvarez P, Eichenbaum H (2005) Neural correlates of social odor recognition and the representation of individual distinctive social odors within entorhinal cortex and ventral subiculum. Neuroscience 130(1):259-274
Rabenau KE, O’Toole JM, Bassi R, Kotanides H, Witte L, Ludwig DL, Pereira DS (2004) DEGA/AMIGO-2, a leucine-rich repeat family member, differentially expressed in human gastric adenocarcinoma: effect on ploidy, chromosomal stability, cell adhesion/migration and tumorigenicity. Oncogene 23:5056-5067

Schubert P, Heinemann U, Kolb R (1986) Differential effect of adenosine on pre- and postsynaptic calcium fluxes. Brain Res 376(2):382-386

Sloviter RS (1989) Calcium-binding protein (calbindin-D28 k) and parvalbumin immunocytochemistry: localization in the rat hippocampus with specific reference to the selective vulnerability of hippocampal neurons to seizure activity. J Comp Neurol 280(2):183-196

Stanfield BB, Cowan WM (1984) An EM autoradiographic study of the hypothalamo-hippocampal projection. Brain Res 309(2):299-307

Stevenson EL, Caldwell HK (2011) The vasopressin 1b receptor and the neural regulation of social behavior. Horm Behav [Epub ahead of print]

Vigers AJ, Baquet ZC, Jones KR (2000) Expression of neurotrophin-3 in the mouse forebrain: insights from a targeted LacZ reporter. J Comp Neurol 416(3):398-415

Winn HR, Welsh JE, Rubio R, Berne RM (1980) Changes in brain adenosine during bicuculline-induced seizures in rats. Effects of hypoxia and altered systemic blood pressure. Circ Res 47(4): 568-577

Young D, Dragunow M (1995) Neuronal injury following electrically induced status epilepticus with and without adenosine receptor antagonism. Exp Neurol 133(2):125-137

Young WS, Li J, Wersinger SR, Palkovits M (2006) The vasopressin $1 \mathrm{~b}$ receptor is prominent in the hippocampal area CA2 where it is unaffected by restraint stress or adrenalectomy. Neuroscience 143(4):1031-1039

Zhao X, Lein ES, He A, Smith SC, Aston C, Gage FH (2001) Transcriptional profiling reveals strict boundaries between hippocampal subregions. J Comp Neurol 441:187-196 